\title{
TheGMS \\ The Gazette of Medical Sciences
}

https://www.thegms.co

ISSN: 2692-4374

DOI: https://www.doi.org/10.46766/thegms

\section{Volume 1 Issue 6 \\ Date of release: 1 January 2021 \\ Frequency: Bi-Monthly (November-December 2020) \\ Issue DOI: https://www.doi.org/10.46766/thegms.v1.i6}

\section{Orthopedics | Retrospective Study}

Study of the efficiency of clinical examination in the diagnosis of meniscal injuries in patients submitted to knee arthroscopy in a University hospital

Thiago Pereira Guimarães ${ }^{1}$, Daniel Estachiote Bastos ${ }^{1}$, Max Rogério Freitas Ramos ${ }^{2 *}$, Flavio Cruz $^{3}$

${ }^{1}$ Orthopaedic Resident by Federal University of the State of Rio de Janeiro UNIRIO

${ }^{2}$ Associate Professor of Orthopedics and Traumatology, Federal University of the Rio de Janeiro State UNIRIO, Head of Orthopedic Clinics at Gaffrée Guinle University Hospital HUGG

${ }^{3}$ Orthopaedic Surgeon and Sports Medicine Doctor, Gaffrée Guinle University Hospital HUGG

Published: 12 November 2020

Pages: 001-012

Cite: Guimarães TP, Bastos DE, Ramos MRF, Cruz F. Study of the efficiency of clinical examination in the diagnosis of meniscal injuries in patients submitted to knee arthroscopy in a University hospital. G Med Sci. 2020; 1(6): 001-012. https://www.doi.org/10.46766/thegms.ortho.20110802

\section{Orthopedics | Prospective cohort study}

The role of Tranexamic Acid in bleeding control during Total Hip Arthroplasty: An analysis

Adrienne de Lemos Basto ${ }^{1}$, Willian Colleti Hagemann ${ }^{1}$, Max Rogério Freitas Ramos ${ }^{2 *}$

${ }^{1}$ Orthopaedic Resident by Federal University of the State of Rio de Janeiro UNIRIO

${ }^{2}$ Associate Professor of Orthopedics and Traumatology, Federal University of the Rio de Janeiro State UNIRIO, Head of Orthopedic Clinics at Gaffrée Guinle University Hospital HUGG

Published: 14 November 2020

Pages: 013-021

Cite: Basto ADL, Hagemann WC, Ramos MRF. The role of Tranexamic Acid in bleeding control during Total Hip Arthroplasty: An analysis. G Med Sci. 2020; 1(6): 013-021.

https://www.doi.org/10.46766/thegms.ortho.2011081 


\section{Neurology | Research article}

\section{A simple immunohistochemical method for detecting microglia in rat brain}

Takashi Ishijima ${ }^{1}$, Kazuyuki Nakajima $^{2 *}$

${ }^{1,2}$ Department of Science and Engineering for Sustainable Innovation, Faculty of Science and Engineering, Soka University, Tokyo 192-8577, Japan.

Published: 11 December 2020

Pages: 022-029

Cite: Ishijima T, Nakajima K. A simple immunohistochemical method for detecting microglia in rat brain. G Med Sci. 2020; 1(6): 022-029. https://www.doi.org/10.46766/thegms.neuro.20120401

\section{Immunology | Research article}

\section{Alzheimer's Disease and COVID-19 fatalities linked to the NLRP3 inflammasome overregulation}

David Macias-Verde ${ }^{1.2^{*}}$, Pedro C. Lara ${ }^{2,3,4}$, Javier Burgos-Burgos ${ }^{2}$

${ }^{1}$ Universidad de Las Palmas de Gran Canaria, Spain.

${ }^{2}$ Oncology Department, Hospitales Universitarios San Roque, Las Palmas de Gran Canaria, Spain.

${ }^{3}$ Universidad Fernando Pessoa Canarias, Las Palmas de Gran Canaria, Spain.

${ }^{4}$ Instituto Canario de Investigación del Cáncer, Tenerife, Spain.

Published: 16 December 2020

Pages: 030-035

Cite: David MV, Lara PC, Javier BB. Alzheimer's and COVID-19 fatalities linked to the NLRP3 inflammasome overregulation. G Med Sci. 2020; 1(6): 030- 035. https://www.doi.org/10.46766/thegms.immuno.20121002

\section{Microbiology | Research article}

A one-year study of bacterial isolates causing Healthcare Acquired Infection (HAI) and their antibiotic resistance pattern at a private hospital in Egypt

Rasha A. Mosbah*

Associate professor of microbiology and immunology, Zagazig University, Egypt. Infection Control Specialist, Infection Control Unit, Zagazig University, Egypt.

Published: 27 December 2020

Pages: 036-045

Cite: Mosbah RA, A one-year study of bacterial isolates causing Healthcare Acquired Infection (HAI) and their antibiotic resistance pattern at a private hospital in Egypt. G Med Sci. 2020; 1(6): 036-045.

https://www.doi.org/10.46766/thegms.microb.20122105 
Neurology | Prospective study

Tau aggregates possibly compromise neuronal health in the C. elegans model of Alzheimer's disease

Sanjib Guha ${ }^{1^{*}}$

${ }^{1}$ University of Rochester, Department of Anesthesiology \& Perioperative Medicine, Rochester, NY

Published: 29 December 2020

Pages: 046-048

Cite: Guha S, Tau aggregates possibly compromise neuronal health in the $C$. elegans model of Alzheimer's disease. G Med Sci. 2020; 1(6): 046-048. https://www.doi.org/10.46766/thegms.neuro.20122004

\section{Neurology | Case report}

\section{Pseudomeningocele after Laminectomy: Case report and literature review}

Nicolle dos Santos Moraes Nunes ${ }^{1}$, Jacqueline Stephanie Fernandes do Nascimento ${ }^{2}$, Juliana Cristina Ferreira Hasegawa $^{3}$, Milena da Costa Araújo Pereira Andrade ${ }^{4}$, Karine Anauha da Silva Toneti ${ }^{5}$, Isabele de Mello Bentes Vaz ${ }^{6}$, Lucas Cruz Ferreira ${ }^{7}$, Marco Orsini ${ }^{8}$, Gilberto Canedo Martins Jr ${ }^{9}$, Antônio Marcos da Silva Catharino ${ }^{10^{*}}$

${ }^{1}$ Undergraduate student of Medicine at the Iguaçu University (UNIG). Nova Iguaçu, RJ, Brazil. https://orcid.org/0000-0003-0336-2261

${ }^{2}$ Undergraduate student of Medicine at the Iguaçu University (UNIG). Nova Iguaçu, RJ, Brazil. https://orcid.org/0000-0003-1847-3829

${ }^{3}$ Undergraduate student of Medicine at the Iguaçu University (UNIG). Nova Iguaçu, RJ, Brazil. https://orcid.org/0000-0003-3129-7006

${ }^{4}$ Undergraduate student of Medicine at the Iguaçu University (UNIG). Nova Iguaçu, RJ, Brazil. ${ }^{5}$ Undergraduate student of Medicine at the Iguaçu University (UNIG). Nova Iguaçu, RJ, Brazil. https://orcid.org/0000-0002-8303-8623

${ }^{6}$ Undergraduate student of Medicine at the Iguaçu University (UNIG). Nova Iguaçu, RJ, Brazil.

${ }^{7}$ Undergraduate in Medicine at the Iguaçu University (UNIG). Nova Iguaçu, RJ, Brazil. https://orcid.org/00000002-6960-9645

${ }^{8}$ Neurologist. Professor of Neurology at the Iguaçu University (UNIG). Nova Iguaçu, RJ, Brazil. Caduceu Institute - Postgraduate Medical Continuing School - SP. https://orcid.org/0000-0001-6680-3795

${ }^{9}$ Neurologist, Professor of Medicine at Universidade Iguaçu, Universidade Iguaçu - UNIG / RJ, Nova Iguaçu, Brazil. https://orcid.org/0000-0002-8181-7930

${ }^{10}$ Neurologist. Professor of Neurology at the Iguaçu University (UNIG). Nova Iguaçu, RJ, Brazil. https://orcid.org/0000-0002-5736-1486

Published: 30 December 2020

Pages: 049-052

Cite: Nunes NSM, Nascimento JSF, Hasegawa JCF, Andrade MCAP, Toneti KAS, Vaz IMB, et al. Pseudomeningocele after Laminectomy: Case report and review. G Med Sci. 2020; 1(6): 049-052. https://www.doi.org/10.46766/thegms.neuro.20122406 


\section{Oncology | Review article}

\section{Cancer management during COVID-19 period-A preliminary study}

Anita Ramesh ${ }^{1}$, Praveen Sahu ${ }^{2}$, Lakshya Mittal ${ }^{2}$, Raji Sundararajan ${ }^{2 *}$, Ignacio Camarillo ${ }^{3}$

${ }^{1}$ Saveetha Medical College \& Hospital, Chennai, India

${ }^{2}$ School of Engineering Technology, Purdue University, West Lafayette, IN 47907- USA

${ }^{3}$ Biological Sciences Department, Purdue University, West Lafayette, IN 47907-USA

Published: 31 December 2020

Pages: 053-068

Cite: Ramesh A, Sahu P, Mittal L, Sundararajan R, Camarillo I. Cancer management during COVID-19 period-A preliminary study. G Med Sci. 2020; 1(6): 053-068. https://www.doi.org/10.46766/thegms.oncol.20122003 